\title{
Conformal GaP layers on Si wire arrays for solar energy applications
}

\author{
Adele C. Tamboli, ${ }^{\text {a) }}$ Manav Malhotra, Gregory M. Kimball, Daniel B. Turner-Evans, and \\ Harry A. Atwater \\ Thomas J. Watson Laboratories of Applied Physics, California Institute of Technology, Pasadena, \\ California 91125, USA
}

(Received 20 June 2010; accepted 10 November 2010; published online 3 December 2010)

\begin{abstract}
We report conformal, epitaxial growth of GaP layers on arrays of Si microwires. Silicon wires grown using chlorosilane chemical vapor deposition were coated with GaP grown by metal-organic chemical vapor deposition. The crystalline quality of conformal, epitaxial $\mathrm{GaP} / \mathrm{Si}$ wire arrays was assessed by transmission electron microscopy and x-ray diffraction. Hall measurements and photoluminescence show $\mathrm{p}$ - and n-type doping with high electron mobility and bright optical emission. GaP pn homojunction diodes on planar reference samples show photovoltaic response with an open circuit voltage of $660 \mathrm{mV}$. (C) 2010 American Institute of Physics.

[doi:10.1063/1.3522895]
\end{abstract}

Microwire arrays are a promising geometry for solar energy conversion in semiconductors with a limited minority carrier diffusion length. ${ }^{1}$ The microwire geometry decouples the absorption length and minority carrier diffusion length, allowing for absorption of nearly all above band gap incident light while enabling efficient carrier collection. ${ }^{2}$ We have previously shown high fidelity synthesis of vertically oriented, high aspect ratio silicon wire arrays using $\mathrm{Cu}-$ catalyzed vapor-liquid-solid growth. ${ }^{3}$ Multijunction wire array solar cells represent a new avenue for attaining higher efficiencies in wire array solar cells than are achievable with single-junction devices. While we have already demonstrated solar cells based on $\mathrm{Si}$ wire arrays, ${ }^{4}$ multijunction cells will require conformal growth of a lattice-matched wider bandgap material, such as GaP or GaNPAs, ${ }^{5}$ on these Si wires. Further, solar fuel generation systems can be envisioned which consist of catalyst-decorated wire arrays as the anode and cathode, separated by an ion-permeable membrane. For these devices, the higher open circuit voltage attainable with larger band gap semiconductors such as GaP allow sufficient photovoltage to drive water splitting reactions. The wire array geometry has the added benefit of high surface area, potentially enabling a high chemical reduction rate even for catalysts with a limited turnover frequency per area. ${ }^{6}$

The wire arrays used in this study range in height from $10-50 \mu \mathrm{m}$ and are 1-2 $\mu \mathrm{m}$ in diameter, as determined by the catalyst particle size during VLS growth and growth time. Prior to GaP growth, we chemically etch the silicon dioxide and copper catalyst and remove a thin surface layer of silicon containing residual $\mathrm{Cu}$ catalyst impurities. To coat these wire arrays with gallium phosphide, we use metalorganic chemical vapor deposition with trimethyl gallium and phosphine precursors. We use a two-step growth procedure with a thin nucleation layer grown at $530{ }^{\circ} \mathrm{C}$ followed by a thick $\mathrm{GaP}$ layer grown at $750{ }^{\circ} \mathrm{C}$. These layers are either p-type or n-type, depending on the V/III ratio during growth: p-type layers are grown using a V/III ratio of 10, and n-type layers are grown using a V/III ratio of 80 . There are no additional dopants added during growth. Reference samples consisting of $\mathrm{GaP}$ grown on planar $\mathrm{Si}$ substrates

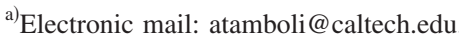

were synthesized along with each set of wire array samples. The thicknesses of the $\mathrm{GaP}$ layers grown on planar Si substrates were 4-5 $\mu \mathrm{m}$, including a $40 \mathrm{~nm}$ nucleation layer, while $\mathrm{GaP}$ grown simultaneously on $\mathrm{Si}$ wire arrays exhibited $200 \mathrm{~nm}$ to $>1 \mu \mathrm{m} \mathrm{GaP}$ coating, depending on the aspect ratio of the $\mathrm{Si}$ wire array used as a growth substrate.

We have characterized the $\mathrm{GaP}$ coated $\mathrm{Si}$ wires using $\mathrm{x}$-ray diffraction, photoluminescence (PL), Hall effect measurements, and both scanning and transmission electron microscopy (SEM, TEM). X-ray diffraction measurements were made in $\omega-2 \theta$ and reciprocal space map configurations. Bright field and high-resolution TEM were performed at an accelerating voltage of $300 \mathrm{kV}$. Samples were prepared for TEM by embedding the wire arrays in epoxy before microtoming $50-100 \mathrm{~nm}$ radial and longitudinal layers. PL measurements were performed using a closed-cycle cryostat cooled to 78 K. Samples were excited using the $458 \mathrm{~nm}$ line of an Ar-ion laser that was chopped at $10 \mathrm{kHz}$ using an acousto-optic modulator. The emission was passed through a monochromator and focused onto a visible frequency chargecoupled detector.

The growth conditions discussed above resulted in conformal coatings of $\mathrm{GaP}$ on $\mathrm{Si}$ microwire arrays, as shown in Fig. 1. Cross-sectional SEM images of cleaved wires reveal

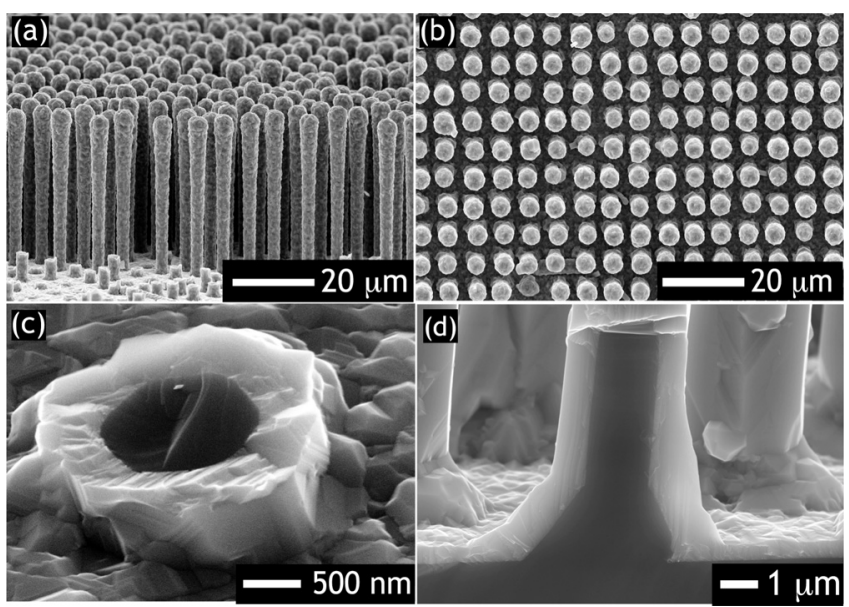

FIG. 1. SEM images of GaP coated Si wire arrays: (a) side view, (b) topdown, and [(c) and (d)] cleaved wires revealing GaP shell and Si core. 


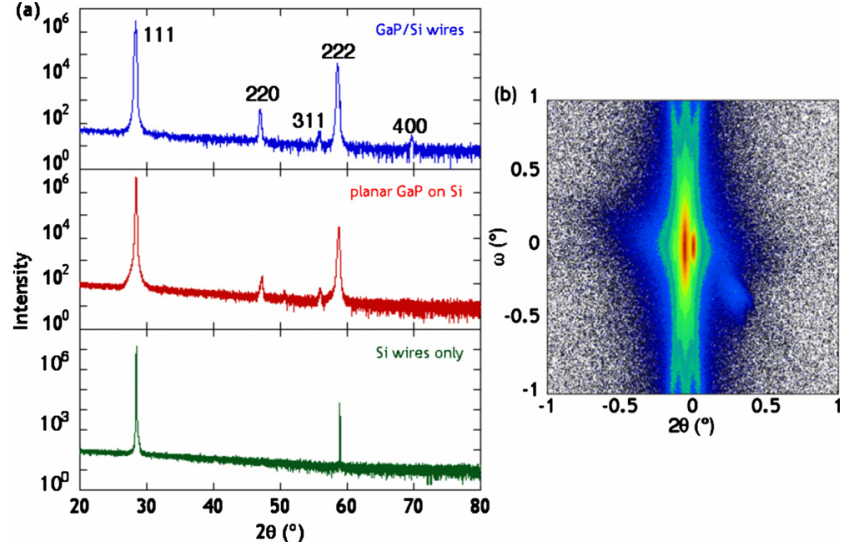

FIG. 2. (Color online) X-ray diffraction measurements: (a) $\omega-2 \theta$ scans of $\mathrm{GaP} / \mathrm{Si}$ wire arrays, planar $\mathrm{GaP}$ on $\mathrm{Si}$ samples from the same growth, and $\mathrm{Si}$ wires without a GaP coating. (b) Reciprocal space map centered around the $\langle 111\rangle$ peak of a GaP/Si wire sample.

that the GaP coating thickness varies along the length of the wires, with the thickest coating at the top of the wires and thinnest on the Si substrate in between wires. This effect is most pronounced in longer wires. The growth morphology of the GaP is typically rough, whether grown on wire arrays or on planar Si substrates included in the same growth run. ${ }^{7}$

$\mathrm{X}$-ray diffraction measurements show that the epitaxial $\mathrm{GaP}$ is preferentially oriented in the $\langle 111\rangle$ direction, matching the orientation of the Si wire array substrates, as shown in Fig. 2. All the planar and wire array GaP/Si samples studied showed preferential orientation in the $\langle 111\rangle$ direction according to $\omega-2 \theta$ scans [Fig. 2(a)], but several samples showed $\langle 220\rangle$ and $\langle 311\rangle$ peaks as well. The non- $\langle 111\rangle$ peaks are more prevalent in the wire array samples relative to the planar GaP/Si samples. Broken off, nonvertically oriented $\mathrm{GaP} / \mathrm{Si}$ wires may contribute to the structural disorder in the $\mathrm{GaP} / \mathrm{Si}$ wire arrays. From these $\omega-2 \theta$ scans, we estimate that on average, phases oriented in non- $\langle 111\rangle$ directions would account for less than $0.05 \%$ of the sample volume if they were uniformly distributed. A reciprocal space map centered around the $\langle 111\rangle$ peak [Fig. 2(b)] from a GaP/Si wire array sample has $\mathrm{Si}$ and $\mathrm{GaP}$ peaks at the predicted locations. The $\mathrm{GaP}$ peak is much broader than the Si substrate peak, with a full width at half maximum of 69.5 and $669 \operatorname{arcsec}$ in the $2 \theta$ and $\omega$ directions, respectively. The narrow peak width in the $2 \theta$ direction indicates a well-oriented film, while the broader peak width in the $\omega$ direction suggests a high defect density, as is typically the case for polar, III-V materials grown on nonpolar substrates.

TEM images and selected-area electron diffraction patterns taken along the $\langle 111\rangle$ zone axis are shown in Fig. 3. TEM imaging shows conformal coating of $\mathrm{GaP}$ on $\mathrm{Si}$ with an

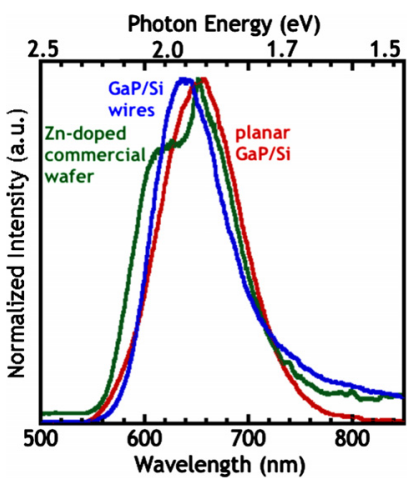

FIG. 4. (Color online) PL spectra from three different types of samples: planar n-type $\mathrm{GaP}$ on $\mathrm{Si}$, an n-type conformally $\mathrm{GaP}$ coated $\mathrm{Si}$ wire sample, and a commercial $\mathrm{Zn}$-doped $\mathrm{GaP}$ wafer.

abrupt interface [Fig. 3(b)]. Diffraction patterns of the microtomed samples show single crystal Si wires [Fig. 3(d)] and single crystal, epitaxial GaP coatings [Fig. 3(e)]. The Si and $\mathrm{GaP}$ diffraction patterns share the same symmetry, but the extra peaks in the GaP diffraction pattern are evidence of twinning. These twin defects may contribute to the broadening of the X-ray spectrum in the $\omega$-direction as well. Using TEM imaging in conjunction with EDS, we found no residual $\mathrm{Cu}$ catalyst in the $\mathrm{Si}$ wires or $\mathrm{GaP}$ coatings from the VLS growth within detection limits, which is consistent with the very long minority carrier diffusion lengths that have been recently measured in silicon wires ${ }^{8}$ and suggests that $\mathrm{Cu}$ is not a significant impurity in the $\mathrm{GaP}$ as well.

Three samples were studied using PL: a GaP coated Si wire array, a planar GaP/Si reference sample, and a commercial Zn-doped GaP wafer obtained from MTI Corporation (Fig. 4). All three samples exhibited broad emission centered around $650 \mathrm{~nm}$. Previous studies ${ }^{9}$ have attributed this subbandgap luminescence to defect states within the bandgap, including $\mathrm{Zn}-\mathrm{O}$ pair recombination, as is visible in the $\mathrm{Zn}$ doped commercial wafer. Dixit et al. ${ }^{10}$ attribute the $1.9 \mathrm{eV}$ emission peak arising from $\mathrm{GaP}$ grown on $\mathrm{Si}$ to donoracceptor pair recombination, where $\mathrm{Si}$ on the $\mathrm{P}$ site is the deep acceptor and a deep donor has not been unambiguously identified. Impurities such as $\mathrm{Zn}, \mathrm{O}, \mathrm{Si}$, and $\mathrm{C}$ could contribute to luminescence in this range. ${ }^{9,11}$

Using planar $\mathrm{GaP} / \mathrm{Si}$ reference samples, we have performed Hall measurements at room temperature using a 1.4 $\mathrm{T}$ electromagnet to determine doping density and mobility. The n-type samples analyzed consisted of $5 \mu \mathrm{m}$ of $\mathrm{GaP}$ grown on an insulating silicon substrate with indium contacts annealed at $400{ }^{\circ} \mathrm{C}$ for $15 \mathrm{~min}$ in forming gas. These samples had typical mobilities of $300 \mathrm{~cm}^{2} / \mathrm{V} \mathrm{s}$ and carrier concentrations of $8 \times 10^{16} \mathrm{~cm}^{-3}$. The p-type samples studied consisted of $1 \mu \mathrm{m}$ of $\mathrm{p}-\mathrm{GaP}$ grown on $4 \mu \mathrm{m}$ of $\mathrm{n}-\mathrm{GaP}$, on
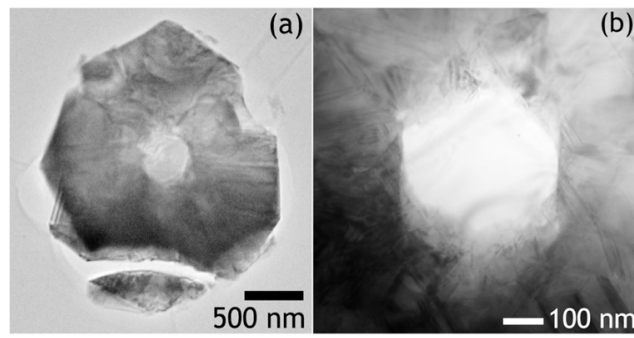

(b)

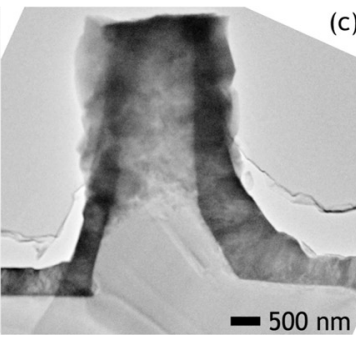

(c)

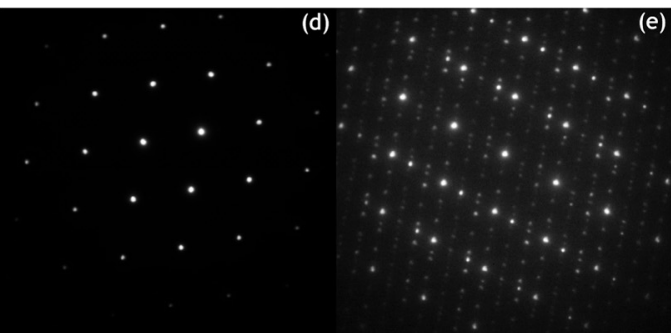

FIG. 3. TEM images (a)-(c) of cross sections of GaP/Si wires. Selected area electron diffraction of Si core (d) and GaP shell (e) taken along the $\langle 111\rangle$ zone axis, showing the shared symmetry of the two lattices and twin defects in the GaP layer. 

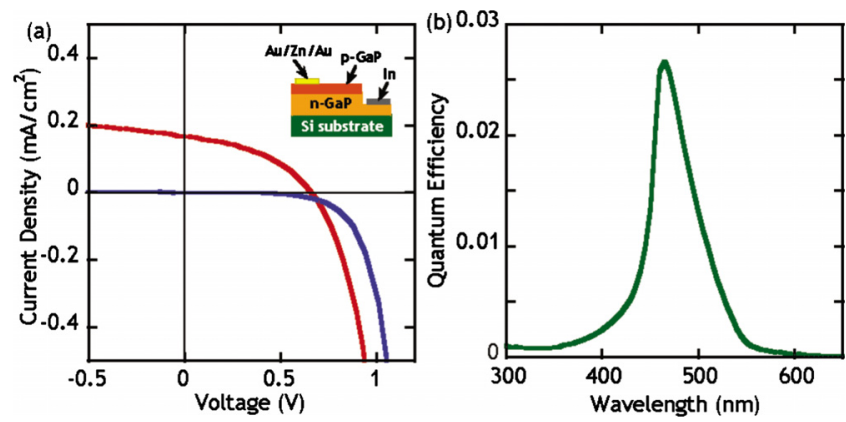

FIG. 5. (Color online) (a) Dark and light IV curves and (b) spectral response for a $\mathrm{GaP}$ homojunction diode on a $\mathrm{Si}$ substrate.

an insulating $\mathrm{Si}$ substrate with $\mathrm{Au} / \mathrm{Zn} / \mathrm{Au} 30 / 30 / 200 \mathrm{~nm}$ contacts. These p-type layers had typical mobilities of $8 \mathrm{~cm}^{2} / \mathrm{V} \mathrm{s}$ and carrier concentrations of $1 \times 10^{18} \mathrm{~cm}^{-3}$. The n-type mobilities measured are consistent with typical single crystal $\mathrm{GaP}$ values for that doping level, ${ }^{12}$ indicating that the presence of twins does not adversely affect carrier transport. The p-type mobility is lower than expected for single crystal $\mathrm{GaP}$ with a high acceptor density, ${ }^{13}$ likely because of the structural disorder induced by changing the V/III ratio so drastically.

Junction properties and photoresponse were studied using planar samples that consisted of $500 \mathrm{~nm}$ of p-type $\mathrm{GaP}$ on $4 \mu \mathrm{m}$ of n-type $\mathrm{GaP}$, on a high resistivity Si substrate. Electrical contacts were made to the n- and p-type layers by etching mesas and depositing In and $\mathrm{Au} / \mathrm{Zn} / \mathrm{Au}$ contacts, as described above [Fig. 5(a), inset]. Dark and light IV curves from these diodes showed rectifying behavior, as shown in Fig. 5(a). Under simulated 1 sun illumination, these diodes exhibited photovoltaic response with an open circuit voltage of $660 \mathrm{mV}$, a short circuit current of $0.17 \mathrm{~mA} / \mathrm{cm}^{2}$, and a fill factor of $42 \%$. The high resistance evident in these IV curves comes from the series resistance of the n-type layer, since only a very thin layer remained after the mesa etch and the doping of this layer was relatively low. Spectral response measurements [Fig. 5(b)] show peak absorption at $460 \mathrm{~nm}$, corresponding to the direct transition in $\mathrm{GaP}$, and falling off gradually toward $550 \mathrm{~nm}$, near the indirect bandgap of GaP. The low photoresponse in the blue region between 300 and $400 \mathrm{~nm}$ is likely due to poor collection efficiency in the p-type region. Both a high surface recombination velocity at the front surface and increased bulk recombination in the low mobility p-type layer can account for the low photoactivity in the near-UV. In the spectral range $450-550 \mathrm{~nm}$, a fit to the spectral response data ${ }^{14}$ using absorption data from Aspnes and Studna ${ }^{5}$ gives an upper bound on the minority carrier diffusion lengths of about $20 \mathrm{~nm}$ in the p- and n-type layers. The same fit gives values of $1.1 \mu \mathrm{m}$ and $140 \mathrm{~nm}$ for junction depth and depletion width, respectively, in agreement with our predictions based on growth and doping. These short diffusion lengths contribute to the low photovoltaic efficiency demonstrated and motivate further work on wire array devices, which are less sensitive to minority carrier diffusion length than planar solar cells. ${ }^{1}$
We have demonstrated growth of conformal layers of $\mathrm{GaP}$ on $\mathrm{Si}$ microwire arrays. X-ray diffraction and TEM analysis show that the GaP films are epitaxial and well oriented, sharing the $\langle 111\rangle$ orientation of the Si wires used as a substrate. Both techniques show evidence of a high defect density, likely twin defects caused by the polar on nonpolar nature of the growth. Twin defects are a common problem in III-V materials grown on group IV substrates and are often minimized by a careful choice of substrate orientation or using offcut substrates. ${ }^{16,17}$ Although the wire array geometry restricts our choice of substrate orientation, wire geometry devices should be more tolerant of defects than planar devices. Despite the structural defects, however, we are able to obtain single crystalline, conformal layers and observe photoluminescence and high electron mobility, which indicate material with good electrical and optical characteristics. We have also demonstrated pn junctions in planar reference samples which show photovoltaic response, giving further evidence of reasonable minority carrier collection. These devices are the first step toward more complex device designs such as multijunction solar cells.

Funding for this work was provided by DARPA and the Caltech Center for Sustainable Energy Research. D.B.T.-E. acknowledges fellowship support from the NSF. The authors thank Nick Strandwitz, Carrie Hofmann, Carol Garland, and Emily Warmann for assistance with GaP processing and characterization

${ }^{1}$ B. M. Kayes, H. A. Atwater, and N. S. Lewis, J. Appl. Phys. 97, 114302 (2005).

${ }^{2}$ M. D. Kelzenberg, S. W. Boettcher, J. A. Petykiewicz, D. B. TurnerEvans, M. C. Putnam, E. L. Warren, J. M. Spurgeon, R. M. Briggs, N. S. Lewis, and H. A. Atwater, Nature Mater. 9, 239 (2010).

${ }^{3}$ B. M. Kayes, M. A. Filler, M. C. Putnam, M. D. Kelzenberg, N. S. Lewis, and H. A. Atwater, Appl. Phys. Lett. 91, 103110 (2007).

${ }^{4}$ M. C. Putnam, S. W. Boettcher, M. D. Kelzenberg, D. B. Turner-Evans, J. M. Spurgeon, E. L. Warren, R. M. Briggs, N. S. Lewis, and H. A. Atwater, Energy Environ. Sci. 3, 1037 (2010).

${ }^{5}$ J. F. Geisz and D. J. Friedman, Semicond. Sci. Technol. 17, 769 (2002).

${ }^{6}$ S. W. Boettcher, J. M. Spurgeon, M. C. Putnam, E. L. Warren, D. B. Turner-Evans, M. D. Kelzenberg, J. R. Maiolo, H. A. Atwater, and N. S. Lewis, Science 327, 185 (2010).

${ }^{7}$ V. K. Dixit, T. Ganguli, T. K. Sharma, S. D. Singh, R. Kumar, S. Porwal, P. Tiwari, A. Ingale, and S. M. Oak, J. Cryst. Growth 310, 3428 (2008).

${ }^{8}$ M. C. Putnam, D. B. Turner-Evans, M. D. Kelzenberg, S. W. Boettcher, N. S. Lewis, and H. A. Atwater, Appl. Phys. Lett. 95, 163116 (2009).

${ }^{9}$ M. Gershenzon, F. A. Trumbore, R. M. Mikulyak, and M. Kowalchik, J. Appl. Phys. 36, 1528 (1965).

${ }^{10}$ V. K. Dixit, T. Ganguli, T. K. Sharma, R. Kumar, S. Porwal, V. Shukla, A. Ingale, P. Tiwari, and A. K. Nath, J. Cryst. Growth 293, 5 (2006).

${ }^{11}$ P. J. Dean, C. J. Frosch, and C. H. Henry, J. Appl. Phys. 39, 5631 (1968).

${ }^{12}$ Y. C. Kao and O. Eknoyan, J. Appl. Phys. 54, 2468 (1983).

${ }^{13}$ H. C. Casey, Jr., F. Ermanis, and K. B. Wolfstirn, J. Appl. Phys. 40, 2945 (1969).

${ }^{14}$ S. M. Sze and K. K. Ng, Physics of Semiconductor Devices, 3rd ed. (Wiley, New Jersey, 2007), pp. 725-730.

${ }^{15}$ D. E. Aspnes and A. A. Studna, Phys. Rev. B 27, 985 (1983).

${ }^{16}$ H. Kroemer, K. J. Polasko, and S. C. Wright, Appl. Phys. Lett. 36, 763 (1980).

${ }^{17}$ H. Kroemer, J. Cryst. Growth 81, 193 (1987). 